\title{
Degradation and Detection of the Nerve Agent VX by a Chromophore Functionalized Zirconium MOF
}

\author{
Martijn C. de Koning ${ }^{\mathrm{a}}$, Gregory W. Peterson ${ }^{\mathrm{b}}$, Marco van Grola ${ }^{\mathrm{a}}$ Ivan Iordanov ${ }^{\mathrm{b}}$, Monica \\ McEntee ${ }^{b}$
}

a) TNO Defense, safety \& Security. Lange Kleiweg 137, 2288GL, Rijswijk, The Netherlands; b) Edgewood Chemical Biological Center, 8198 Blackhawk Road, Aberdeen Proving Ground, MD, United States

\section{SUPPORTING INFORMATION}

\section{General information}

\section{Equipment}

All compounds and solvents were used as received from the suppliers (Sigma-Aldrich). Analytical LC was conducted on a Waters HPLC-system (515 HPLC pump, 2545 binary gradient modulator, System fluidics Organizer, and a 2998 photodiode array detector) using an Alltima C18 analytical column ( $5 \mu$ particle size, flow: $1.0 \mathrm{ml} / \mathrm{min})$. The system was connected to a Waters Acquity QDA detector (mass spectrometer) that could operate in both positive and negative ion modes. ${ }^{1} \mathrm{H}-\mathrm{NMR},{ }^{13} \mathrm{C}-\mathrm{NMR}$ and ${ }^{31} \mathrm{P}-\mathrm{NMR}$ spectra were recorded on a Bruker Avance III spectrometer operating at $400 \mathrm{MHz}, 100 \mathrm{MHz}$ and $162 \mathrm{MHz}$ respectively. Chemical shifts $(\delta)$ are given in ppm relative to tetramethyl silane $(\delta 0 \mathrm{ppm})$, or to residual solvent signals. $\mathrm{N}_{2}$ isotherms were measured on a Micromeritics instrument (ASAP2020). PXRD measurements were conducted using a Bruker D8 or a Rigaku Miniflex 600 benchtop instrument. UV-VIS spectra were recorded on a JASCO V-650 spectrophotometer. DRIFTS Equipment and Procedure: The diffuse reflectance infrared Fourier transform spectroscopy (DRIFTS) system consists of a Harrick Praying Mantis ${ }^{\mathrm{TM}}$ Diffuse Reflection Accessory connected to the internal compartment of a Thermo Nicolet 6700 FTIR spectrometer. Each sample (NU-1000, DTNB, DTNB@NU-1000-1, and DTNB@NU-1000-2) was loosely packed into a 3 mm diameter stainless steel cup and transferred to the Harrick cell. Once inside the cell, each sample was purged for $\sim 20$ minutes in $\mathrm{CO}_{2}$ - and $\mathrm{H}_{2} \mathrm{O}$-free air, and the FTIR spectra were collected with an 
average of 256 interferograms per spectrum at a resolution of $2 \mathrm{~cm}^{-1}$. The background spectrum was collected using $\mathrm{KBr}$ powder with the same parameters and experimental conditions as the samples.

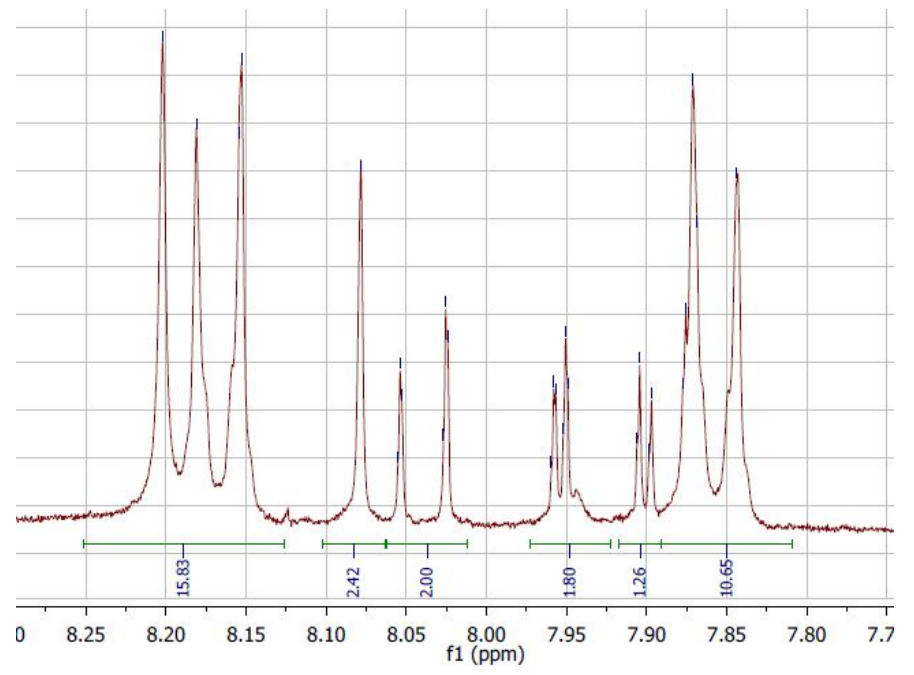

Figure S1. Part of the NMR spectrum of a digested DTNB@NU-1000 sample. The integrals of the 3 peaks between 8.22-8.12 ppm (linker, $12 \mathrm{H}$ ) and of the 2 peaks between 8.06-8.02 ppm (DTNB, 2H) were used to calculate the loading.

\section{DTNB@NU-1000 PXRD}

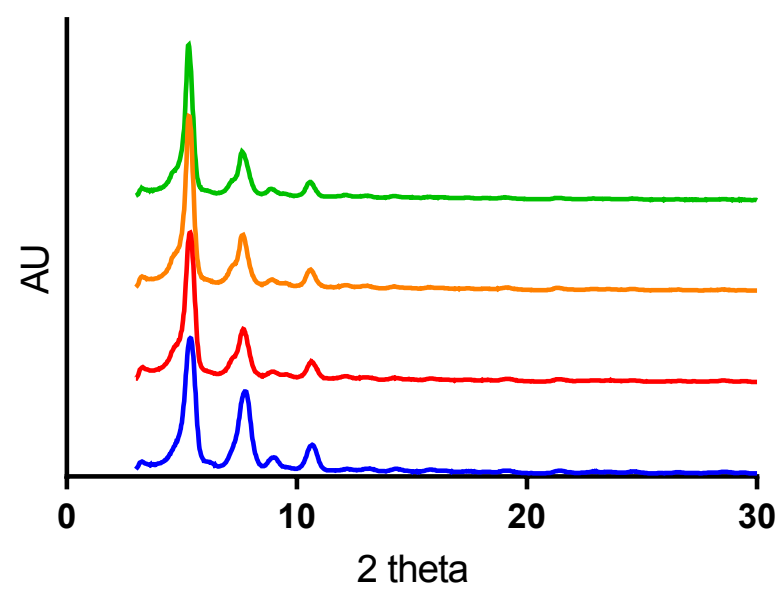

— DTNB@NU-1000-0.4

— DTNB@NU-1000-1

— DTNB@NU-1000-2

- NU-1000 reference 
Figure S2. PXRD spectra of NU-1000 (blue) and NU-1000 loaded with various amounts of $\operatorname{DTNB}(0.4,1 \& 2 \mathrm{~mol}$ DTNB / mol NU-1000; compared to (activated) NU-1000.

\section{$\mathbf{N}_{2}$ adsorption isotherms}

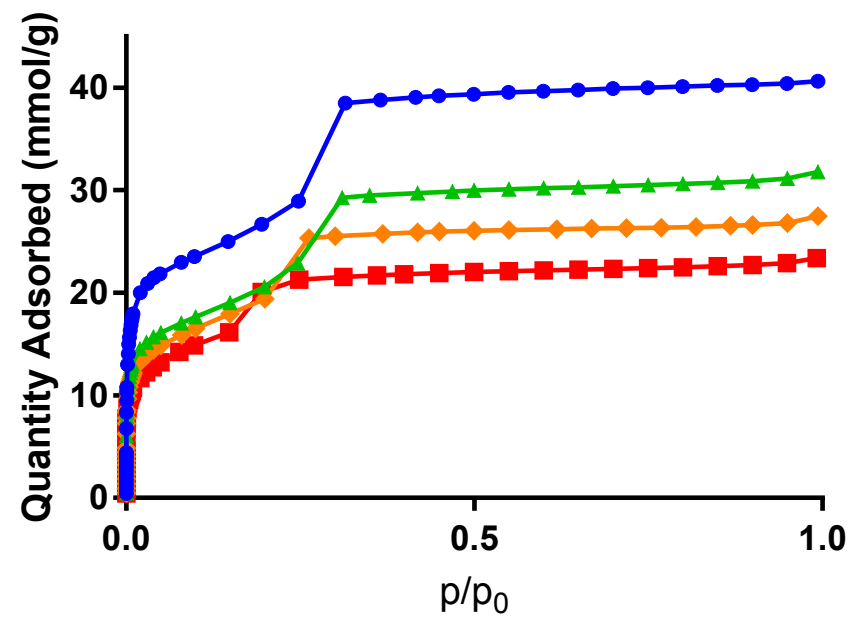

$\rightarrow$ NU-1000 $\rightarrow$ DTNB@NU-1000-1

=DTNB@NU-1000-0.4 - DTNB@NU-1000-2

Figure S3. $\mathrm{N}_{2}$ adsorption isotherms of NU-1000 (blue) and NU-1000 loaded with various amounts of DTNB@NU-1000-\#. Loading (\#) is given in mole DTNB per mole Zr $\mathrm{r}_{6}$-SBU.

Table S1. Brunauer-Emmett-Teller (BET) surface areas and pore volumes of a number of DTNB-loaded NU-1000 samples.

\begin{tabular}{|c|c|c|}
\hline $\begin{array}{c}\text { DTNB@NU-1000 } \\
(\mathrm{mol} / \mathrm{mol})\end{array}$ & $\begin{array}{l}\text { BET surface area } \\
\qquad\left(\mathrm{m}^{2} / \mathrm{g}\right)\end{array}$ & $\begin{array}{l}\text { Total pore volume } \\
\left(\mathrm{cm}^{3} / \mathrm{g}\right)\end{array}$ \\
\hline 0 & 2140 & 1.33 \\
\hline 0.4 & 1638 & 1.02 \\
\hline 1 & 1560 & 0.88 \\
\hline 2 & 1393 & 0.75 \\
\hline
\end{tabular}




\section{Binding mode modeling of DTNB in NU-1000 and DRIFTS spectra of DTNB@NU-1000-2}

Knowing that each $\mathrm{Zr}_{6}$ SBU can host a maximum of four carboxylic acid functional groups, two possible binding modes of DTNB may be postulated to explain the maximum occupancy of 2 mole of DTNB per mole SBU.

One explanation would require regioselective binding of two molecules of DTNB, via one of its carboxylic acids, to two easily accessible coordination sites on each $\mathrm{Zr}_{6}$ SBU while leaving the remaining two sites, that may be sterically constrained ${ }^{[1]}$, unaffected. This binding mode could be excluded after a failed attempt to occupy the remaining open binding sites in DTNB@NU-10002 by a SALI treatment with a large excess of DTNB, as well as by a secondary SALI treatment with the small-sized benzoic acid. Instead of an increase in occupied sites, this treatment merely resulted in partial replacement of DTNB by benzoic acid molecules.

Thus, DTNB molecules chelate with both of their carboxylic acid groups to the same SBU. Molecular modeling suggested that such a binding mode of DTNB is feasible (Figure S4). However, molecular modeling suggested that, in order to avoid major distortion in the structural integrity of the MOF, one carboxylic acid binds with both oxygens to the SBU, while the other carboxylic acid chelates with a single oxygen to the second coordination site.
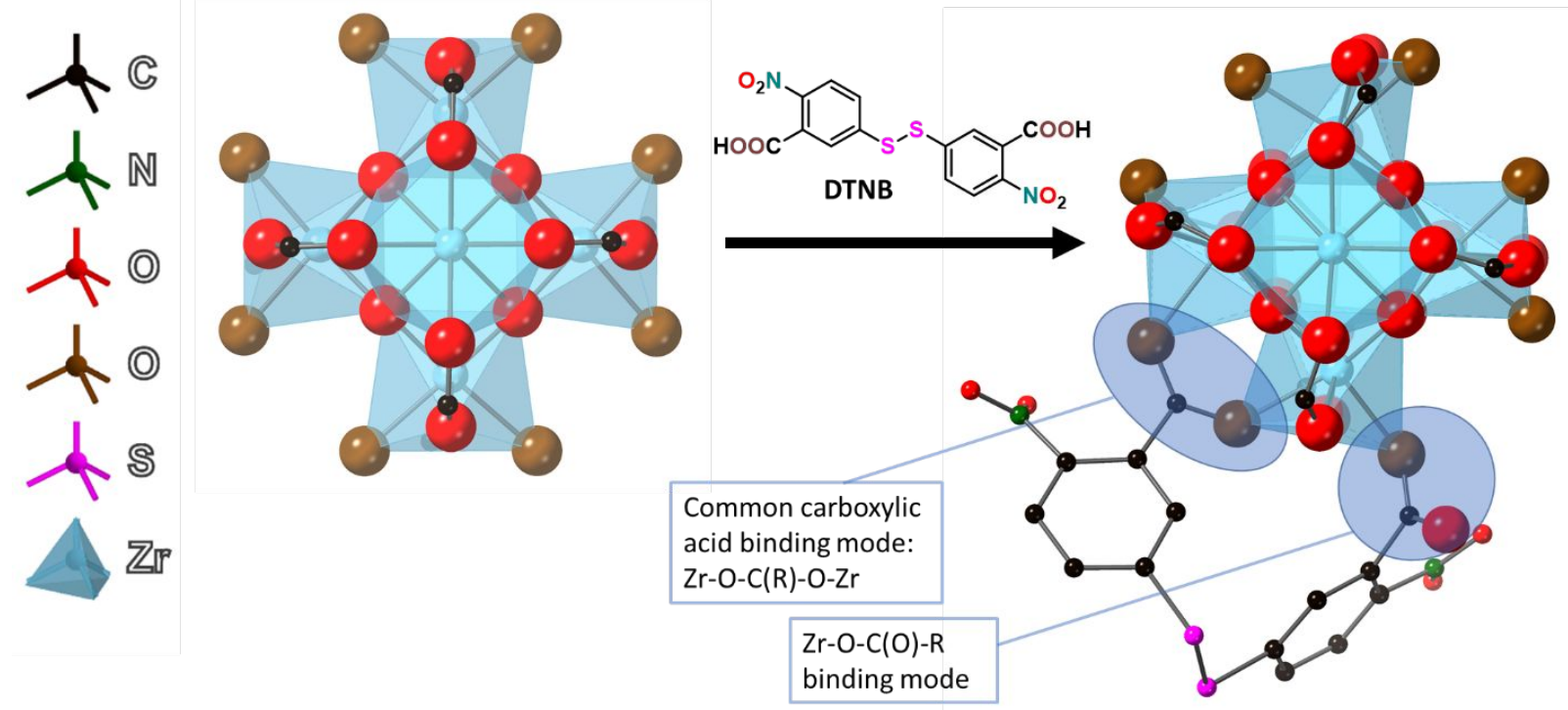
Figure S4. Calculated model of the binding mode of DTNB in NU-1000 (displayed for 1 of the 2 $D T N B$ s binding to the $S B U$ ). One carboxylic acid binds with both oxygen atoms coordinated to zirconium, while the other carboxylic acid chelates its OH group only.

DRIFTS was used to verify this postulated binding mode. The spectra (Figure S5) showed, as expected, a decrease of the $\mathrm{Zr}-\mathrm{OH}$ absorption bands $\left(\sim 3675 \mathrm{~cm}^{-1}\right)$ with increasing DTNB content. The spectra further showed several different types of $\mathrm{C}=\mathrm{O}$ bands: $\sim 1710-1740 \mathrm{~cm}^{-1}$ was assigned to $\mathrm{COOH}$ for DTNB alone (as shown by the DTNB standard in green) and NU-1000 linker alone (black curve), $\sim 1670 \mathrm{~cm}^{-1}$ was assigned to $\mathrm{C}=\mathrm{O}$, and $1600-1630 \mathrm{~cm}^{-1}$ and $1400-1460$ $\mathrm{cm}^{-1}$ were both assigned to asymmetric and symmetric stretches, respectively, of $\mathrm{COO}$ with both $\mathrm{C}-\mathrm{O}$ bound to the zirconium. Following the trend that more binding occurs with decreasing wavenumber, then the peak at $\sim 1670 \mathrm{~cm}^{-1}$ could be assigned a $\mathrm{C}=\mathrm{O}$ where the $\mathrm{OH}$ dissociated to bind to zirconium leaving the $\mathrm{C}=\mathrm{O}$ unbound. The intensity of this band increases with increasing DTNB content, and broadens which may indicate slightly different strengths due to the strain of the DTNB molecule itself on the surface.
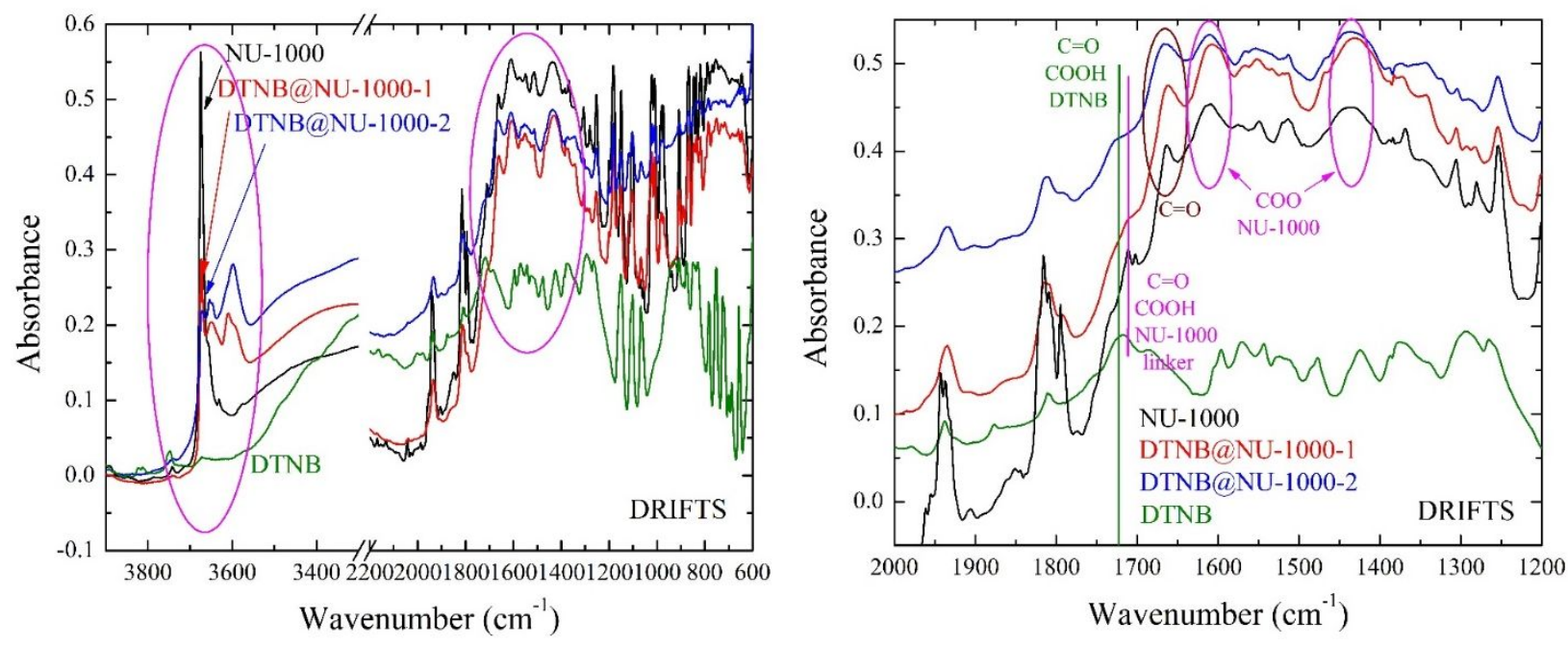
Figure S5. DRIFTS spectra of activated NU-1000 (black), DTNB (green), DTNB@NU-1000-1 (red) and DTNB@NU-1000-2 (blue) to reveal the different C=O absorptions.

\section{Stability of DTNB@NU-1000-2 in various buffers over time}

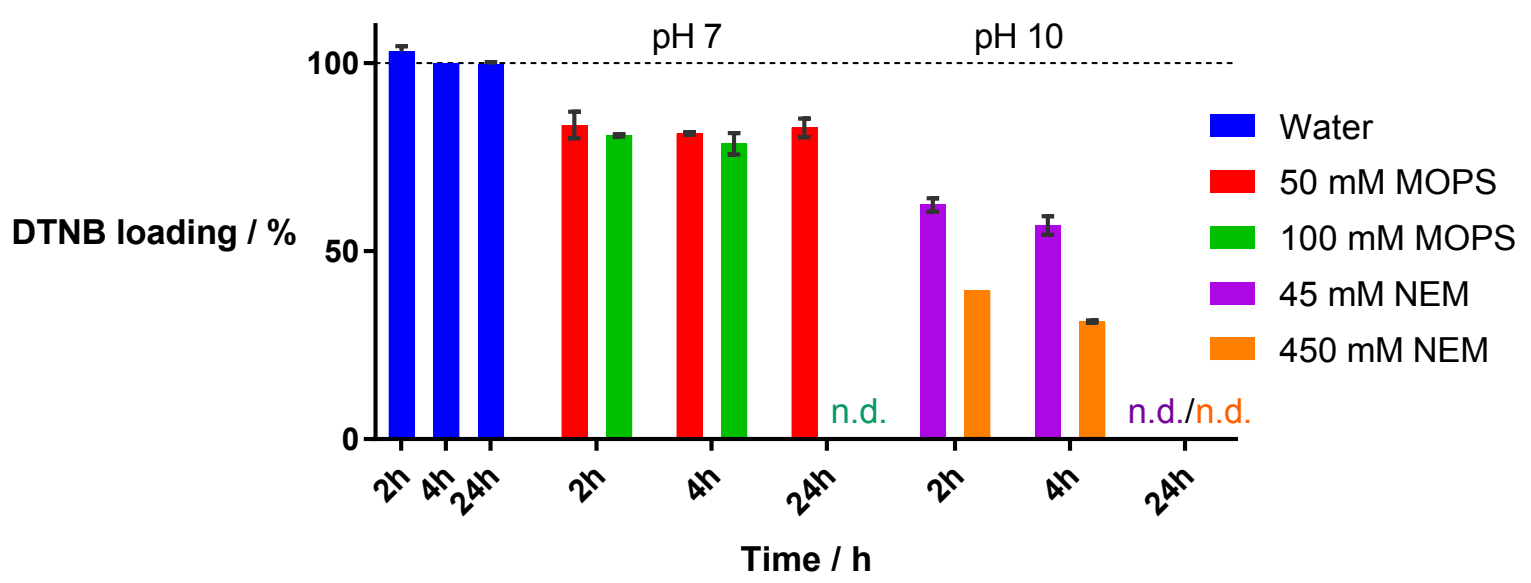

Figure S6. DTNB loading after exposure of DTNB@NU-1000-2 to various buffers for various times.

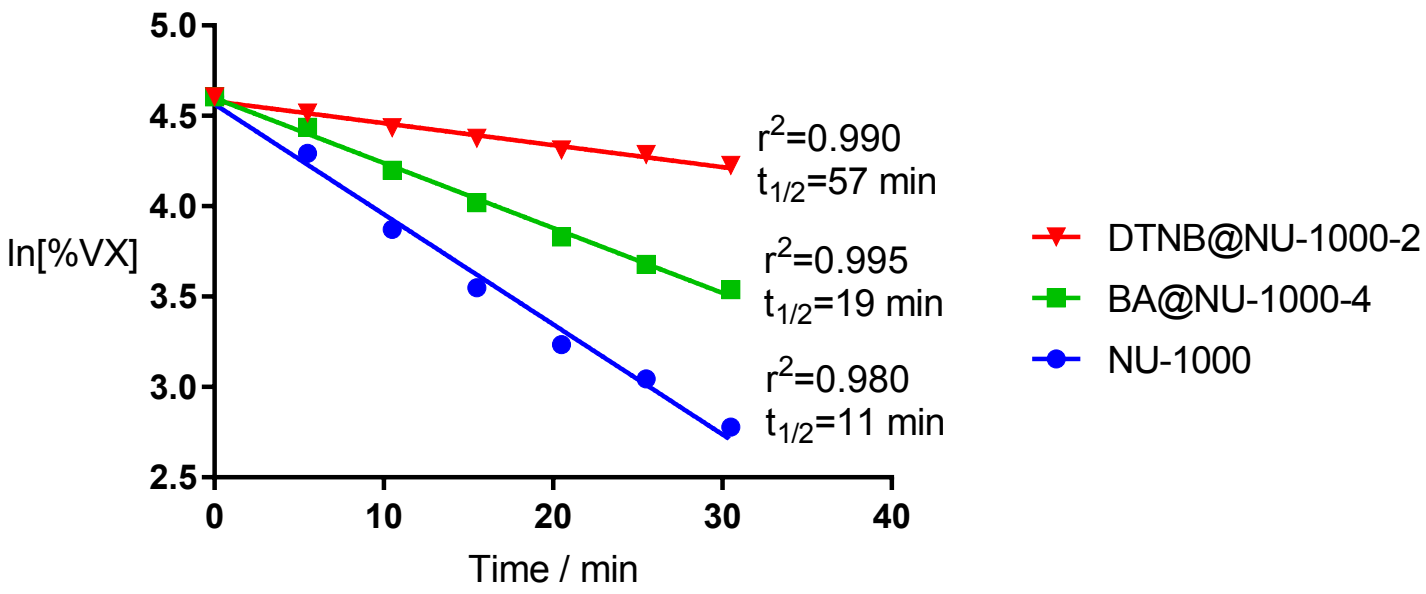


Figure S7. Determination of the first order initial rates of the degradation of VX $(25 \mathrm{mM}$ in $1 \mathrm{ml}$ 50 mM MOPS buffer pH 7) by equimolar amounts of NU-1000, DTNB@NU1000-2 and BA@NU-1000-4. (BA=benzoic acid; BA@NU-1000-4 is the product of the NU-1000 synthesis, prior to acid-mediated activation).

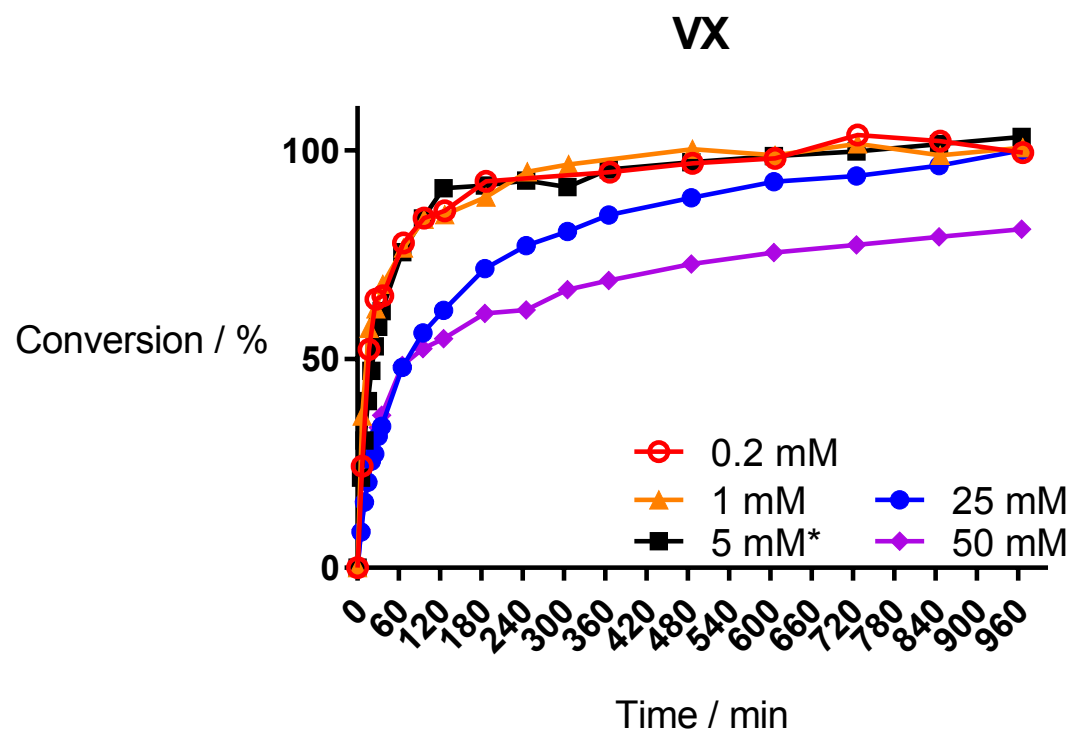

Figure S8. Degradation curves of various concentrations of VX in MOPS buffer (50 mM, pH 7), mediated by DTNB@NU-1000-2. 


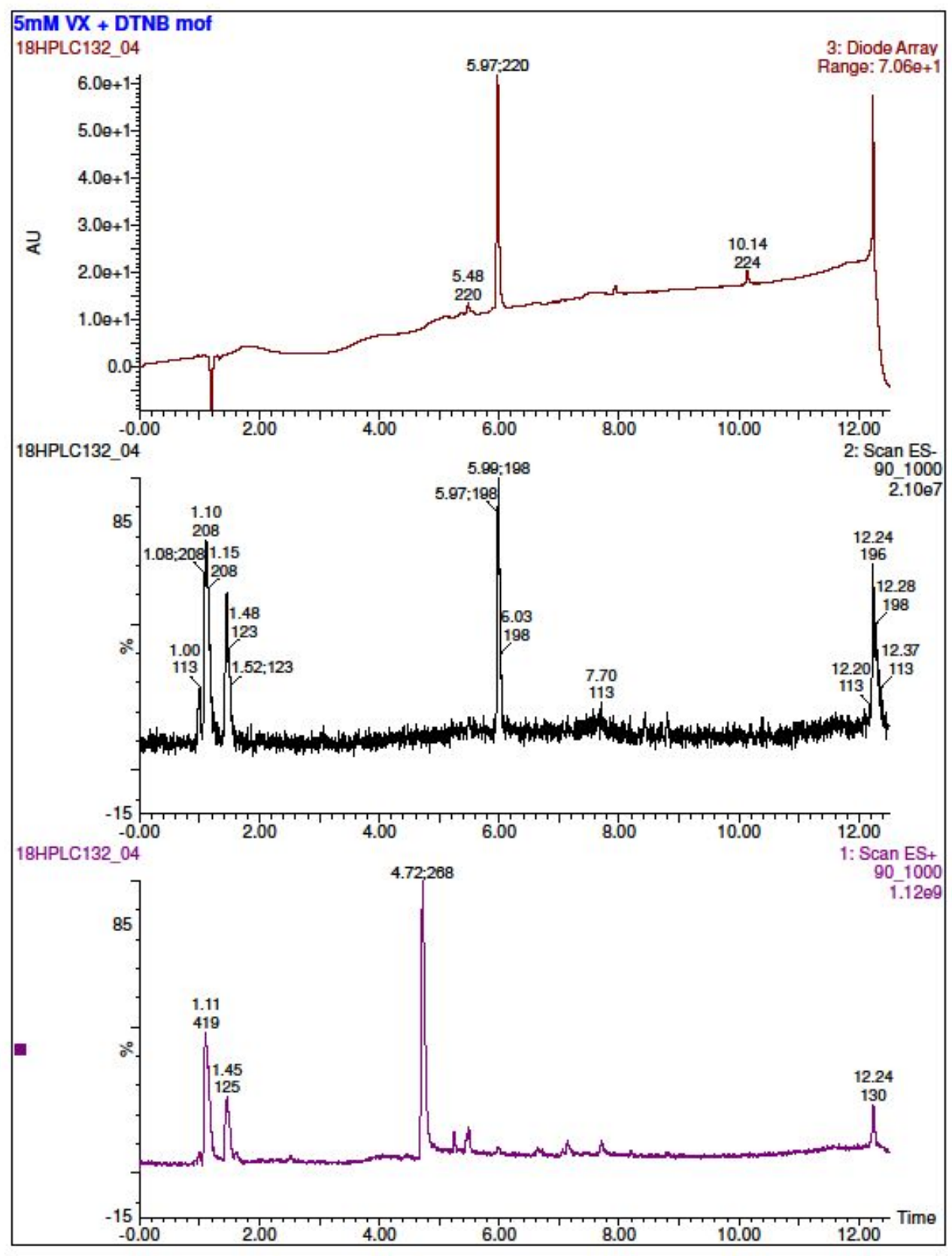

Figure S9. LC-MS analysis of the filtrate of the 15 minute reaction between VX (5 mM) and DTNB@NU-1000-2 in MOPS buffer. Top: UV trace; middle: Mass Spectrometry trace in negative ion mode; bottom: Mass spectrometry trace in positive ion mode. Main products: Injection peak (1.10 min): MOPS ([M-H $\left.]^{-}=208\right) ; 1.48 \mathrm{~min}:$ EMPA $\left([\mathrm{M}-\mathrm{H}]^{-}=123\right) ; 4.72 \mathrm{~min}$ : $\operatorname{VX}\left([\mathrm{M}+\mathrm{H}]^{+}=268\right) ; \mathrm{t}=5.97 \mathrm{~min}: \mathrm{TNB}\left([\mathrm{M}-\mathrm{H}]^{-}=198\right)$. 


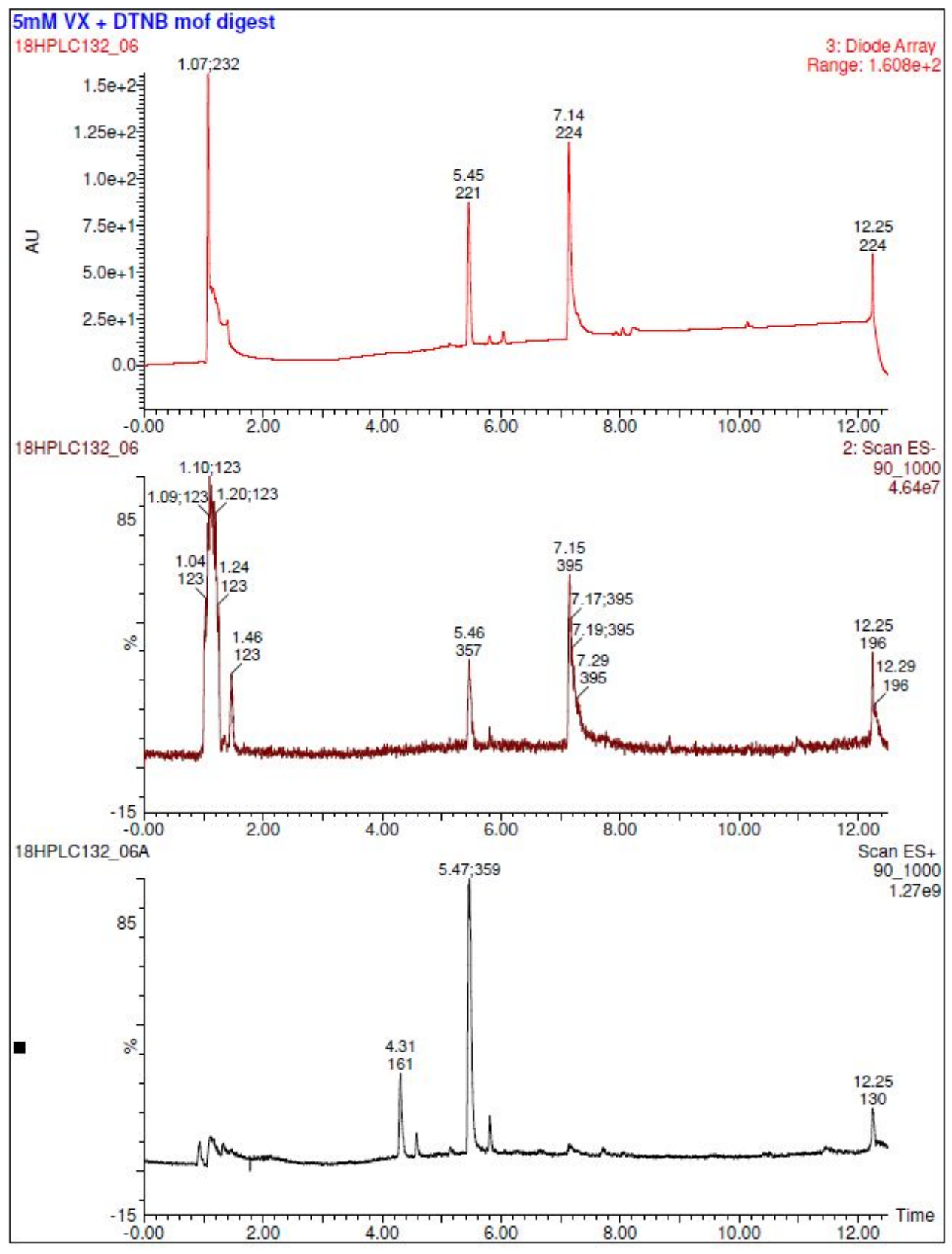

Figure S10. LC-MS analysis of the digest of the MOF obtained after filtration of the 15 minute reaction between VX (5 mM) and DTNB@NU-1000-2 in MOPS buffer. Top: UV trace; middle: Mass Spectrometry trace in negative ion mode; bottom: Mass spectrometry trace in positive ion mode. Main products: 1.10 min: EMPA $(\mathrm{M}-\mathrm{H}=123)$; 4.3 min: DESH dimer $\left([\mathrm{M}+2 \mathrm{H}]^{2+}=161\right)$; $\mathrm{t}=5.45$ min: TNB-DESH $\left([\mathrm{M}+\mathrm{H}]^{+}=359 ;[\mathrm{M}-\mathrm{H}]^{-}=357\right) ; 7.14 \min : \operatorname{DTNB}\left([\mathrm{M}-\mathrm{H}]^{-}=395\right)$. 


\section{Determination visual detection limit}

10 DTNB samples with varying absorption levels (created by addition of various amounts of DESH to a solution of DTNB in MOPS buffer) were prepared (including a blanc). The samples were coded in random order and showed twice to 10 different persons in two separate rounds. The persons were asked to indicate which samples they would confidently denote as 'positive' (a visual yellow coloration compared to the blanc). They did not know they were shown the same samples twice as they were differently coded, so duplicate 'measurements' were obtained for each person.

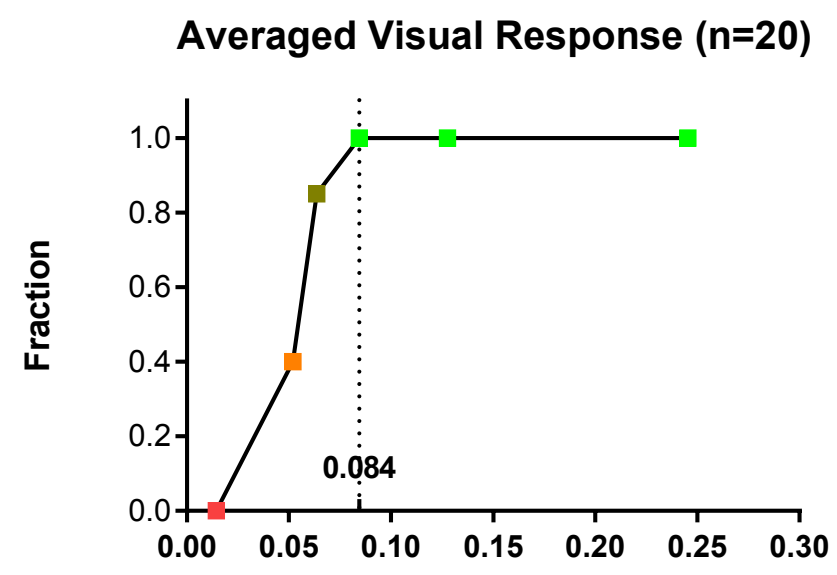

Absorption (AU)

Figure S11. Fraction of positive judgements upon comparison of several test samples with varying coloration (absorption, $\mathrm{x}$-axis) with a blank sample. The visual limit of detection was defined as the concentration for which $100 \%$ positive judgements were made.

\section{References}

[1] J. E. Mondloch, W. Bury, D. Fairen-Jimenez, S. Kwon, E. J. DeMarco, M. H. Weston, A. A. Sarjeant, S. T. Nguyen, P. C. Stair, R. Q. Snurr, et al., J. Am. Chem. Soc. 2013, 135, 10294-10297. 